EESTI NSV TEADUSTE AKADEEMIA TOIMETISED

FUOSIKA * MATEMAATIKA

ИЗВЕСТИЯ АКАДЕМИИ НАУК ЭСТОНСКОП ССР. ФИЗИКА * МАТЕМАТИКА

PROCEEDINGS OF THE ACADEMY OF SCIENCES OF THE ESTONIAN SSR.

PHYSICS * MATHEMATICS

$1986,35,3$

УДК 512.534

В. ФЛЯИШЕР

\title{
О СПЛЕТЕНИЯХ МОНОИДОВ С КАТЕГОРИЯМИ
}

\author{
(Представил А. Хумал)
}

Конструкция сплетений играет важную роль в теории групп [ $\left.{ }^{1}\right]$, теории полугрупп $[2,3]$, теории автоматов $\left[{ }^{4,5}\right]$. Большое значение имеет она при изучении полугрупп эндоморфизмов свободных полигонов $\left[{ }^{6-8}\right]$. Так, Л. А. Скорняков $\left[{ }^{8}\right]$ получил теорему, которая при определенных предположениях дает необходимые условия изоморфизма сплетений моноидов и обобщает результат определяемости свободного полигона своей полугруппой эндоморфизмов [ $\left.{ }^{9}\right]$.

В настоящей статье описывается более общая конструкция сплетения моноида с малой категорией и рассматриваются вопросы изоморфизма гакого сплетения. Для различных классов категорий получены необходимыс и достаточные условия изоморфизма таких сплетений, причем в качестве одного из следствий в предположениях теоремы Л. А. Скорнякова $\left[{ }^{8}\right]$ получены необходимые и достаточные условия изоморфизма сплетений моноидов. Другим следствием из полученных здесь результагов является известная ранее основная теорема $\left[{ }^{10}\right]$.

Пусть $\Omega$ - произвольная малая категория с множеством объектов $X=\mathrm{Ob} \Omega$ и иножеством морфизмов $M=$ Mor $\Omega$. Для произвольных $x, y \in X$ через $M(x, y)$ будем обозначать множество морфизмов из объекта. $x$ в объект $y$. В дальнейшем, обозначая произведение морфизмов $\alpha \beta$, будем предполагать, что вначале применяется морфизм $\beta$. Для любого $x \in X$ через $M(x)$ обозначим множество морфизмов, исходящих из $x$, т.е. $M(x)=U M(x, y)$. Пусть $S$ произвольный моноид и $X=\mathrm{Ob} \Omega$ есть левый $S$-полигон. Через $F(X, M)$ будем обозначать совокупность всех таких отображений $f: X \rightarrow M$, что для любого $x \in X$ выполняется $f(x) \in M(x)$. В множестве $S \times F(X, M)$ рассмотрим подмножество $\mathfrak{A}$, состоящее из всех таких пар $(s, f)$, что для любого $x \in X$ выполняется $f(: z) \in M(x, s x)$, т. e.

$$
\mathfrak{U}=\{(s, f) \mid s \in S, f \in F(X, M), \forall x \in X f(x) \in M(x, s x)\} .
$$

На множестве $\mathfrak{A}$ определим умножение следующим образом: для любых $(s, f),(t, g) \in \mathfrak{U}$

$$
(s, f)(t, g)=\left(s t, f_{t} g\right),
$$

где $(f, g)(x)=f(t x) g(x) \quad$ для любого $x \in X$. Легко проверить, что множество $\mathfrak{A}$ относительно операции умножения, определенной формулой (1); является полугруппой. Эту полугруппу $\mathfrak{A}$ будем называть cnлетением моноида $S$ с категорией $\Omega$ и обозначать $(S$ wr $\AA)$.

Заметим, что сплетение моноида с категорией является более общей конструкцией, чем сплетение двух моноидов. Действительно, всякое сплетение $(S$ wr $R \mid B)$ моноидов $S$ и $R$ с помощью левого $S$-полигона $B$ мы можем рассматривать как сплетение $(S w r \Omega)$ моноида $S$ с категорией $\Omega$, объекты которой суть циклические свободные правые $R$-полигоны 
$b R(b \in B)$, а морфизмы - произвольные $R$-гомоморфизмы. Однако сплетение моноида с категорией не может рассматриваться как частный случай сплетения двух категорий $\left[{ }^{11}\right]$, являясь лишь иногда некоторым объектом такой категории. Заметим также, что сплетение моноида с категорией обобщает полугруппу эндоморфизмов не только свободного, но и проективного полигона над моноидом.

Левый $S$-полигон $X$ называется допустимым, если

1) $|X| \geqslant 2$

2) если $s x=x$ для любого $x \in X$, то $s=1$;

3) для каждого $x \in X$ существует единственный элемент $s \in S$ такой, что $s y=x$ для любого $y \in X$ (такой элемент $s$ будем обозначать $v_{x}$ );

4) для любых $s, t \in S$ и $x, y \in X \quad(x \neq y)$ существует $r \in S$ такой, что $r x=s x, r y=t y$.

Определение допустимого $S$-полигона дано Л. А. Скорняковым $\left[{ }^{8}\right]$. Заметим, что условие 4) в этом определении может быть заменено равносильным ему условием 2-плотности полигона $X$, т. е. условием

$\left.4^{\prime}\right)$ для любых $x, y, a, b \in X \quad(x \neq y)$ найдется $r \in S$ такой, что $r x=a$, $r y=b$.

Действительно, ясно, что 4') влечет 4). Наоборот, по условию 3) имеем $v_{a} x=a, v_{b} y=b$ и значит по условию 4) найдется $r \in S$ такой, что $r x=a, r y=b$,

Л. А. Скорняковым $\left[{ }^{8}\right]$ доказана

Т е о рем а 1. Если $A$ и В допустимые левые полигоны над моноидами $R$ и $S$ соответственно, $U$ и $V$ моноиды $и$

$$
(R \operatorname{wr} U \mid A) \cong(S \operatorname{wr} V \mid B),
$$

то $|A|=|B| u U \cong V$.

В настоящей статье мы для различных классов категорий дадим необходимые и достаточные условия изоморфизма сплетений категорий с моноидами. В качестве следствия мы получим, что в предположениях теоремы 1

$$
(R \operatorname{wr} U \mid A) \cong(S \operatorname{wr} V \mid B)
$$

тогда и только тогда, когда $|A|=|B|, U \cong V, R \cong S$.

Малую категорию $\Omega$ назовем допустимой для моноида $S$, если $\Omega$ - сильно связанная категория, т. е. $M(x, y) \neq \varnothing$ для любых $x, y \in$ $\in X=\mathrm{Ob} \Omega$, и $X$ есть допустимый $S$-полигон. В дальнейшем мы будем рассматривать сплетения моноидов лишь с допустимыми для них категориями.

Пусть $S-$ моноид, $\Omega$ - произвольная допустимая для $S$ малая кагегория и $\mathfrak{A}=(S w r \Re)$. Для любого $x \in X=\mathrm{Ob} \Omega$ через.$_{x}$ обозначим множество всех пар $(s, f) \in \mathcal{H}$, в которых $s=v_{x}$. Пусть $\mathfrak{Y}=\bigcup_{x \in X} \mathfrak{\mathcal { F }}_{x}$. Непосредственно проверкой доказывается следующая

Л е м м а 1. Множество $\mathfrak{9}$ является идеалом в полугруппе $\mathfrak{A}$, представимым в виде объединения попарно-непересекающихся правых идеалов $\mathfrak{\vartheta}_{x}(x \in X)$, порожденных идемпотентами $\left(v_{x}, f\right)$, где $f(x)=1_{x}-$ тождественный морфизм.

Пусть $\mathscr{B}$ - произвольное множество в полугруппе 2 . Через $B(x, y)$ обозначим множество всех морфизмов $\alpha \in M(x, y)$, для которых найдется $(s, f) \in \mathfrak{B}$ так, что $s x=y$ и $f(x)=\alpha$, т. е.

$$
\mathfrak{B}(x, y)=\{f(x) \mid(s, f) \in \mathfrak{B}, s x=y\} .
$$

Л е м м а 2. Пусть $\mathfrak{B}$ - правый идеал в $\mathscr{H}$, порожденный идемпотентом $(s, f)$. Если для некоторых $x, y \in X$ выполняется $\mathfrak{B}(x, y) \neq \varnothing$, то 
$\mathfrak{B}(y, y) \neq \varnothing$ и $\mathfrak{3}(y, y)$ является правым идеалом в $M(y, y)$, порожденным идемпотентом $f(y)$.

Доказ а тельст в о. Пусть $\mathfrak{B}(x, y) \neq \varnothing$. Тогда найдется элемент $(u, g) \in \mathfrak{B}$ такой, что $u x=y$ и значит $g(x) \in \mathfrak{B}(x, y)$. Так как $\mathfrak{B}$ порождается идемпотентом $(s, f)$, то $(u, g)=(s, f)(u, g)=(s u, f u g)$, т. е. $s u=u, f_{u} g=g$. Отсюда следует $s y=s(u x)=(s u) x=u x=y \quad$ и, значит, $f(y) \in \mathfrak{B}(y, y)$. Непосредственной проверкой легко показать, что $\mathfrak{B}(y, y)$ есть правый идеал в $M(y, y)$.

Пусть $\beta$ - произвольный морфизм из $\mathfrak{B}(y, y)$, т. е. $\beta=h(y)$, где $(v, h) \in B$ и $v y=y$. Из $(v, h)=(s, f)(v, h)=\left(s v, f_{v} h\right) \quad$ следует $h=f_{v} h$ и, значит,

$$
\beta=h(y)=\left(f_{v} h\right)(y)=f(v y) h(y)=f(y) \beta .
$$

Таким образом, $f(y)$ есть левая единица в $\mathcal{B}(y, y)$. Лемма доказана.

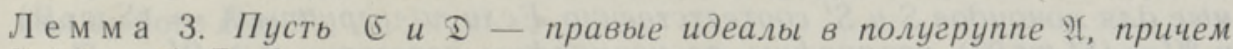
$\leftarrow \cap \mathbb{D}=\varnothing$. Тогда

$$
\mathfrak{E}(x, y) \cap \mathfrak{D}(x, y)=\varnothing
$$

для любых $x, y \in X$.

Д ок а зательство. Предположим противное, пусть найдется $\alpha \in$ $\in M(x, y)$ такой, что $\alpha \in \mathbb{E}(x, y) \cap \mathcal{D}(x, y)$. Тогда существуют $(s, f) \in$ $\Subset \mathbb{E}$ и $(u, g) \in \mathcal{D}$ такие, что $f(x)=g(x)=\alpha$ и, значит, $s x=u x=y$. Отсюда для произвольного $\left(v_{x}, h\right) \in \mathcal{\imath}$ получаем

$$
\begin{gathered}
(s, f)\left(v_{x}, h\right)=\left(s v_{x}, f_{v_{x}} h\right)=\left(v_{y}, f_{v_{x}} h\right) \in \mathcal{E}, \\
(u, g)\left(v_{x}, h\right)=\left(u v_{x}, g_{v_{x}} h\right)=\left(v_{y}, g_{v_{x}} h\right) \in \mathcal{D},
\end{gathered}
$$

причем

$$
\left(f_{v_{x}} h\right)(z)=f(x) h(z)=g(x) h(z)=\left(g_{v_{x}} h\right)(z)
$$

для каждого $z \in X$. Следовательно, $\left.\left(v_{y}, f_{v_{x}} h\right)=v_{y}, g_{v_{x}} h\right) \in \mathfrak{C} \cap D$, что противоречит предположению.

Л е м м а 4. Пусть идеал D в полугруппе $\mathfrak{A}$ представим в виде объединения попарно-непересекающихся правых идеалов $D_{j}(j \in J)$, порожденных идемпотентами $\left(s_{j}, f_{j}\right)$, причем $|J|>1$. Тогда $\mathscr{D} \subseteq 5$.

Д ок а з а тельство. Предположим противное, т. е. $\mathfrak{D} \not \mathscr{g}$. Тогда в $D$ найдется элемент $(u, g)$ такой, что $u x \neq u y$ для некоторых $x, y \in X$. Пусть $(u, g) \in\left(s_{j}, f_{j}\right) \cdot \mathscr{H}$ при некотором $j \in J$. Тогда $\left(s_{j}, f_{j}\right)(u, g)=(u, g)$, откуда следует $s_{j} u=u$. Отсюда вытекает, что $s_{j} u x=u x, s_{j} u y=u y$ и, значит, $f_{j}(u x) \in M(u x, u x), f_{j}(u y) \in M(u y, u y)$. Ввиду 2-плотности $S$-полигона $X$, для произвольного $z \in X$ найдется такой $v \in S$, что $v(u x)=z$, $v(u y)=u y$. Пусть $(v, h)$ такой элемент из $\mathscr{A}$, что $h(u y)=1_{u y}$. Ввиду $(v, h)\left(s_{j}, f_{j}\right)=\left(v s_{j}, h_{s_{j}} f_{j}\right) \in \mathfrak{D}, \quad$ имеем $\left(v_{s_{j}}, h_{s_{j}} f_{j}\right) \in \mathfrak{D}_{i} \quad$ при некотором $i \in J$. Из равенств

$$
\left(h_{s}, f_{j}\right)(u y)=h\left(s_{j} u y\right) f_{j}(u y)=h(u y) f_{j}(u y)=1_{u y} \cdot f_{j}(u y)=f_{j}(u y)
$$

следует, что $f_{j}(u y) \in \mathscr{D}_{j}(u y, u y) \cap \mathscr{D}_{i}(u y, u y)$. Тогда по лемме 3 получаем $\mathfrak{D}_{i} \cap \mathfrak{D}_{j} \neq \varnothing$, т. е. $i=j$. С другой стороны,

$$
\left(h_{s_{j}} f_{j}\right)(u x)=h\left(s_{j} u x\right) f_{j}(u x)=h(u x) f_{j}(u x) \in M(u x, z),
$$

поскольку $v(u x)=z$, т. е. $\mathscr{D}_{j}(u x, z) \neq \varnothing$. По лемме 2 отсюда сльдует, что $\mathfrak{D}_{j}(z, z) \neq \varnothing$ и $\mathfrak{D}_{j}(z, z)$ есть правый идеал в $M(z, z)$, порожденный идемпотентом $f(z)$. Из того, что $f_{j}(z) \in M(z, z)$, мы получаем $s_{j} z=z$ для любого $z \in X$, т. е. $s_{j}=1 \in S$. 
Пусть теперь $k \neq j(k \in J)$, тогда по условию $\mathfrak{D}_{k} \cap \mathfrak{D}_{j}=\varnothing, \mathfrak{D}_{k}=$ $=\left(s_{k}, f_{k}\right) \mathfrak{A},\left(s_{k}, f_{k}\right)\left(s_{k}, f_{k}\right)=\left(s_{k}, f_{k}\right)$. Пусть $x$ - произвольный элемент из $X$ и $y=s_{k} x$, т. е. $s_{h} y=s_{k} s_{k} x=s_{k} x=y$. Пусть $z-$ произвольный элемент из $X$ такой, что $z \neq y$, и пусть $v$ такой элемент из $S$, что $v z=z, v y=y$. Рассмотрим элемент $(v, h) \in \mathscr{U}$ такой, что $h(y)=$ $=f_{k}(y), h(z)=f_{j}(z)$. Ясно, что $(v, h)\left(s_{j}, f_{j}\right)=(v, h)\left(1, f_{j}\right)=\left(v, h f_{j}\right) \in \mathfrak{D}$. Из равенств $\left(h f_{j}\right)(z)=h(z) f_{j}(z)=f_{j}(z) f_{j}(z)=f_{j}(z)$ следует $\left(h f_{j}\right)(z) \in$ $\in \mathfrak{D}_{j}(z, z)$. Это означает, что $\left(v, h f_{j}\right) \in D_{j}$. С другой стороны, $\left(h f_{j}\right)(y)=$ $=h(y) f_{j}(y)=f_{k}(y) f_{j}(y) \in \mathfrak{D}_{k}(y, y), \quad$ т. е. $\left(v, h f_{j}\right) \in \mathfrak{D}_{k}$. Следовательно, $\mathfrak{D}_{k} \cap \mathfrak{D}_{j} \neq \varnothing$, что противоречит выбору $k \neq j$. Лемма доказана.

С л ед с т и е 1. В полугруппе श्ष идеал के является наибольшим идеалом, представимым в виде объединения попарно-непересекающцхся правых идеалов, порсжденных идемпотентами.

Т е о р ем а 2. Пусть $\Omega$ и $\Omega^{\prime}-$ произвольные малые категории, допустимые для моноидов $S$ и $S^{\prime}$ соответственно. Если полугруппы $\mathfrak{A}=($ wr $\mathfrak{i})$ и $\mathfrak{U}^{\prime}=\left(S^{\prime}\right.$ wr $\left.\mathfrak{\Re}^{\prime}\right)$ изоморфны, то изоморфны категории $\mathfrak{R}$ и $\mathfrak{\Re}^{\prime}$.

Д ок а 3 а т ел ь с т о. Пусть $\theta: \mathscr{H} \rightarrow \mathscr{P}^{\prime}$ есть изоморфизм. Тогда по след-

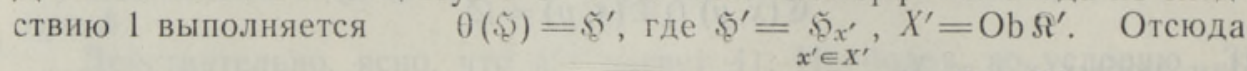
вытекает, что существует биективное отображение $\Phi: X \rightarrow X^{\prime}$ такое, что

для любого $x \in X$.

$$
\theta\left(\mathscr{\mathcal { Y }}_{x}\right)=\mathfrak{S}_{\mathscr{\Phi}(x)}
$$

Пусть $\alpha \in M(x, y)$ - произвольный морфизм в $\Omega$ и пусть $(s, f)$ произвольный элемент из чt такой, что $s x=y$ и $f(x)=\alpha$. Обозначим $\theta((s, f))=\left(s^{\prime}, f^{\prime}\right)$ и положим

$$
\Phi(\alpha)=f^{\prime}(\Phi(x)) \text {. }
$$

Проверим, что отображение $\Phi: \Omega_{i} \rightarrow \mathfrak{\imath}^{\prime}$, определяемое формулами (2) и (3), осуществляет изоморфизм категорий $s i$ и $s i^{\prime}$. Вначале покажем, что отображение $(:) \rightarrow \varsigma^{\prime}$, задаваемое формулой $(3)$, определено корректно. Действительно, пусть $(u, g)$ - другой элемент из $\mathfrak{A}$, для которого $u x=y, g(x)=\alpha$, и пусть $\theta((u, g))=\left(u^{\prime}, g^{\prime}\right)$. Докажем, что $g^{\prime}(\Phi(x))=f^{\prime}(\Phi(x))$.

Для любого $\left(v_{x}, h\right) \in 2$ выполняется

$$
(s, f)\left(v_{x}, h\right)=\left(v_{y}, f_{v_{x}} h\right)=\left(v_{y}, g_{v_{x}} h\right)=(u, g)\left(v_{x}, h\right),
$$

поскольку $\left.\left(f_{v_{x}} h\right)(z)=f(x) h(z)=g(x) h(z)=g_{v_{x}} h\right)(z)$ для любого $z \in X$. Таким образом, $(s, f) \bar{a}=(u, g) \bar{a}$ для любого $\bar{a} \in \mathcal{S}_{x}$. Применяя к этому равенству изоморфизм $\theta$, получим $\left(s^{\prime}, f^{\prime}\right) \bar{a}^{\prime}=\left(u^{\prime}, g^{\prime}\right) \bar{a}^{\prime}$ для любого $\bar{a}^{\prime} \in \mathscr{\bigotimes}_{\Phi(x)}$. Возьмем $\bar{a}^{\prime}=\left(v_{\Phi(x)}, h^{\prime}\right)$, где $h^{\prime}(\Phi(x))=1_{\Phi(x)}$, и тогда необходимо $f^{\prime} v_{\Phi(x)} h^{\prime}=g^{\prime} v_{\Phi(x)} h^{\prime}$, что влечет

$$
\begin{gathered}
f^{\prime}(\Phi(x))=f^{\prime}(\Phi(x)) h^{\prime}(\Phi(x))=\left(f^{\prime} v_{\Phi(x)} h^{\prime}\right)(\Phi(x))= \\
=\left(g^{\prime} v_{\Phi(x)} h^{\prime}\right)(\Phi(x))=g^{\prime}(\Phi(x)),
\end{gathered}
$$

т. е. $f^{\prime}(\Phi(x))=g^{\prime}(\Phi(x))$. Следовательно, отображение (3) определено корректно.

Заметим, что из $s x=y$ следует $(s, f) \vec{a} \in \tilde{\vartheta}_{y}$ для любого $\vec{a} \in \bar{\vartheta}_{x}$. От сюда вытекает, что $\left(s^{\prime}, f^{\prime}\right) \bar{a}^{\prime} \in \mathscr{\xi}_{\Phi(y)}$ для любого $\bar{a}^{\prime} \in \mathscr{\bigvee}_{\Phi(x)}$. А это означает, что

$$
s^{\prime} \Phi(x)=\Phi(y)=\Phi(s x),
$$

т. е. $f^{\prime}(\Phi(x)) \in M(\Phi(x), \Phi(y))$ или $\Phi(\alpha) \in M(\Phi(x), \Phi(y))$.

Остается проверить, что Ф есть функтор, осуществляющий изоморфизм категорий $\Omega$ и $\Omega^{\prime}$. Это проводится непосредственно. 
3 а меча н и е 1. Из теоремы 2 вытекает основной результат статьи $\left(\left[{ }^{10}\right]\right.$, теорема 1). Для этого надо заметить, что если $S$ есть полугруппа всех преобразований множества $X=\mathrm{Ob} \Omega$, то $(S w r \Omega)$ есть в точности полугруппа $S(\Re)$, индуцированная категорией $\Omega\left(\right.$ см. $\left.\left[{ }^{10}\right]\right)$.

Пусть $\Phi: \Omega \rightarrow \Omega^{\prime}$ изоморфизм категорий и $f \in F(X, M)$. 'Через $\Phi(f): X^{\prime} \rightarrow M^{\prime}$, где $X^{\prime}=\mathrm{Ob} \Omega^{\prime} . M^{\prime}=$ Mor $\Omega^{\prime}$ обозначим такое отображение, что $\Phi(f)\left(x^{\prime}\right)=\Phi\left(f\left(\Phi^{-1}\left(x^{\prime}\right)\right)\right)$ для любого $x^{\prime} \in X^{\prime}$, т. е. $\Phi(f)=$ $=\Phi f \Phi^{-1}$. Ясно, что $\Phi(f)\left(x^{\prime}\right) \in M\left(x^{\prime}\right)$ для любого $x^{\prime} \in X^{\prime}$, т. е. $\Phi(f) \in$ $\in F\left(X^{\prime}, M^{\prime}\right)$.

3 а ме ч ан ие 2 . Из доказательства теоремы 2 (см. формулы (3) и (4)) следует, что для любого $(s, f) \in \mathscr{V}$ из $\theta((s, f))=\left(s^{\prime}, f^{\prime}\right)$ вытекает

$$
\begin{gathered}
f^{\prime}=\Phi(f)=\Phi f \Phi^{-1}, \\
s^{\prime} \Phi(x)=\Phi(s x)
\end{gathered}
$$

для любого $x \in X$.

Полигон $X$ над моноидом $S$ называется точным, если для произвольных $s, t \in S$ из $s x=t x$ при каждом $x \in X$ следует $s=t$. Мы скажем. что категория $\Omega$ удовлетворяет правому (левому) условию Оре, если для любых $\alpha, \beta \in M(x, y)$ при произвольных $x, y \in \mathrm{Ob} \Omega$ выполняется $\alpha M(x, x) \cap \beta M(x, x) \neq \varnothing(M(y, y) \alpha \cap M(y, y) \beta \neq \varnothing)$. Например, категория с нулевыми морфизмами, очевидно, удовлетворяет как правому, так и левому условию Оре. Скажем, что категория $\Omega$ есть категория с главными морфизмами, если для любых $x, y \in \mathrm{Ob} \Re$ существует морфизм $e_{x, y} \in M(x, y)$ так, что $M(x, y)=e_{x, y} M(x, x)$.

Т е орем а 3. Пусть $\Omega$ и $\Omega^{\prime}-$ произвольные малые категории, допустимые для моноидов $S$ и $S^{\prime}$ соответственно, и пусть выполнено одно из следующих трех условии:

1) $X=\mathrm{Ob} \Re$ и $X^{\prime}=\mathrm{Ob} \Re^{\prime}$ суть точные полигоны над моноидами $S$ и $S^{\prime}$ соответственно,

2) $\Omega$ и $\Omega^{\prime}$ удовлетворяют правому (левому) условию Оре;

3) $\Omega, \Omega^{\prime}-$ категории с главными морфизмами.

Тогда $(S$ w $\Re) \cong\left(S^{\prime}\right.$ шr $\left.\Omega^{\prime}\right)$ в том и только том случае, если $S \cong S^{\prime}$, $\Re \cong \Omega^{\prime}$. Причем для любого изоморфизма $\theta:(S$ w $\Re) \rightarrow\left(S^{\prime} w r \Omega^{\prime}\right)$ найдутся изоморфизмы $\Phi: \Omega \rightarrow \Re^{\prime}, \varphi: S \rightarrow S^{\prime} \quad$ так, что

а) $\Phi(s x)=\varphi(s) \Phi(x) \quad$ для любых $s \in S, x \in X=\mathrm{Ob} \Omega$,

б) $\theta((s, f))=\left(\varphi(s), \Phi f \Phi^{-1}\right)$ для любого $(s, f) \in(S w r \Omega)$.

Доказ ательство. Пусть $s$ - произвольный элемент из $S$ и $f-$ любой элемент из $F(X, M)$ так, что $(s, f) \in \mathfrak{U}=(S$ wr $\Re)$ и пусть $\theta((s, f))=\left(s^{\prime}, f^{\prime}\right)$. Положим

$$
\varphi(s)=s^{\prime} .
$$

На основании теоремы 2 и замечания 2 наша теорема будет доказана, если мы покажем, что определенное так отображение $\varphi: S \rightarrow S^{\prime}$ является изоморфизмом. Покажем корректность этого определения отдельно в каждом из трех случаев, сформулированных в условии теоремы. Предположим, что $\theta((s, g))=\left(s^{\prime \prime}, g^{\prime}\right)$ для некоторого $(s, g) \in \mathfrak{H}$ и покажем, qто $s^{\prime}=s^{\prime \prime}$.

1) Пусть $X$ и $X^{\prime}$ - точные полигоны. Тогда по замечанию 2 выполняется $s^{\prime} \Phi(x)=\Phi(s x)=s^{\prime \prime} \Phi(x)$ для любого $x \in X$, т. е. $s^{\prime} x^{\prime}=s^{\prime \prime} x^{\prime}$ при любом $x^{\prime} \in X^{\prime}$. Ввиду точности $S^{\prime}$-полигона $X^{\prime}$, следует что, $s^{\prime}=s^{\prime \prime}$.

2) Пусть $\Re$ и $\Omega^{\prime}$ удовлетворяют правому условию Оре. Тогда для любого $x \in X$ найдутся $\alpha_{x}, \beta_{x} \in M(x, x)$ так, что $f(x) \alpha_{x}=g(x) \beta$. Рассмотрим элементы $(1, \alpha),(1, \beta) \in \mathfrak{N}$, где $\alpha(x)=\alpha x, \beta(x)=\beta_{x}$ для каждого $x \in X$. Тогда $(s, f)(1, \alpha)=(s, g)(1, \beta)$, поскольку $(f \alpha)(x)=(g \beta)(x)$. Заметим, 
что $\theta((1, \alpha))=\left(1^{\prime}, \alpha^{\prime}\right)$, где $1^{\prime}$ - единица мононда $S^{\prime}$ и $\alpha^{\prime}=\Phi(\alpha)$, поскольку

$$
1^{\prime} \cdot \Phi(x)=\Phi(1 \cdot x)=\Phi(x)
$$

для любого $x \in X$, т. е. $1^{\prime} x^{\prime}=x^{\prime}$ для каждого $x^{\prime} \in X^{\prime}$. Анало:ично $\theta((1, \beta))=\left(1^{\prime}, \beta^{\prime}\right)$. Следовательно,

$$
\left(s^{\prime}, f^{\prime}\right)\left(1^{\prime}, \alpha^{\prime}\right)=\left(s^{\prime \prime}, g^{\prime}\right)\left(1^{\prime}, \beta^{\prime}\right),
$$

откуда вытекает $s^{\prime}=s^{\prime \prime}$.

При предположении левого условия Оре доказательство проводится аналогично.

3) Пусть $\Omega, \Omega^{\prime}-$ категории с главными морфизмами. Тогда для любого $x \in X$ найдутся $\alpha_{x}, \beta_{x} \in M(x, x)$ такие, что $f(x)=e_{x, s x} \alpha x, g(x)=e_{x, s x} \beta_{x}$ Рассмотрим элемент $(s, e) \in \mathfrak{H}$, где $e(x)=e_{x, s x}$ для каждого $x \in X$ и $(1, \alpha) \in \mathcal{U}$, причем $\alpha(x)=\alpha_{x}$ при любом $x \in X$. Тогда $(s, e)(1, \alpha)=$ $=(s, e \alpha)=(s, f)$ и аналогично $(s, e)(1, \beta)=(s, g)$, где $\beta(x)=\beta_{x}$ для любого $x \in X$. Пусть $\theta((s, e))=\left(t^{\prime}, e^{\prime}\right)$, тогда

$$
\left(t^{\prime}, e^{\prime}\right)\left(1^{\prime}, \alpha^{\prime}\right)=\left(s^{\prime}, f^{\prime}\right), \quad\left(t^{\prime}, e^{\prime}\right)\left(1^{\prime}, \beta^{\prime}\right)=\left(s^{\prime \prime}, g^{\prime}\right),
$$

откуда вытекает $s^{\prime}=t^{\prime}=s^{\prime \prime}$.

Таким образом, отображение $\varphi: S \rightarrow S^{\prime}$ определено корректно. Проверка того, что $\varphi-$ изоморфизм, тривиальна. Теорема доказана.

Қак было отмечено в начале статьи, сплетение произвольных моноидов $(S$ wr $R \mid B)$ с помощью левого $S$-полигона $B$ можно рассматривать как сплетение $(S w r \Omega)$, где $\Omega-$ категория правых $R$-полигонов, объекты которой суть циклические свободные полигоны $b R(b \in B)$, а морфизмы произвольные $R$-гомоморфизмы. Заметим; что такая категория $\Omega$ является категорией с главными морфизмами, поскольку для любых $a R, b R \in$ $\in \mathrm{Ob} \Omega$ выполняется $M(a R, b R)=e_{a, b} M(a R, a R)$, где $e_{a, b}(a r)=b r$ для любого $r \in R$. Таким образом, из теоремы 3 вытскает

Т е о р е а 4. Если $A$ и $B$ допустимые левые полигоны над моноидами $R u S$ соответственно, $U$ u $V-$ моноиды. то $(R w r U \mid A) \cong(S w r V \mid B)$ гогда и только тогда, когда $|A|=|B|, U \cong V, R \cong S$.

Для случая сплетения моноидов теорема 3 дает также возможность описать общий вид всякого изоморфизма сплетений моноидов.

\section{Л И ТЕ Р А Т Р А}

1. Нейман X. Многообразия групп. М., «Мир», 1969.

2. Hunter, R. P. Bull. Soc. Math. Belg., 18, 3-16 (1966).

3. Neumann, B. H. J. London Math. Soc., 35, 184-192 (1960)

4. Алгебраическая теория автоматов, языков и полугрупп (Ред. Арбиб М. А.). М., «Статистика», 1975.

5. Eilenberg, S. Automata, languages, and machines, vol. B. New York-London, Academic Press, 1976.

6. Knauer, U., Mikhalev, A. Semigroup Forum, 19, 177-187, 189-198, 355-369 (1980).

7. Skornjakov, L. A. Semigroup Forum, 18, 83-86 (1979).

8. Skornjakov, L. A. Universal algebra and applications. Banach Center publ., vol. 9. Warsaw, 1982, $181-185$.

9. Фляйщер В.Г. Уч. зап. Тартуск. ун-та, вып. 366, 27-41 (1975).

10. Фляйщер B. Г. Уч. зап. Тартуск. ун-та, вып. 700, $83-89$ (1985).

11. Wells, C. J. Algebra, 64, 37-45 (1980).

Тартуский государственный университет 


\section{MONOIDIDE JA KATEGOORIATE POIMIKUIST}

Artiklis on antud monoidi ja kategooria põimiku konstruktsioon, mis üldistab monoidipõimikuid ja vabade vôi projektiivsete polügoonide endomorfismide poolrühmi. Erinevate kategooriaklasside korral tōestatakse, et üks monoidi ja kategooria põimik on isomorfne teise monoidi ja kategooria pōimikuga ainult siis, kui monoidid ja ka kategooriad on omavahel isomorfsed. Järeldusena on saadud kahe monoidipõimiku isomorfismi tarvilikud ja piisavad tingimused; see kinnitab L. A. Skornjakovi põhitulemust [ $\left.{ }^{8}\right]$.

\section{FLYAISHER}

\section{ON THE WREATH PRODUCT OF MONOIDS WITH CATEGORIES}

A construction of the wreath product of monoids with categories is given. Let $\Omega$ be a small category with $X=\mathrm{Ob} \Omega, M=$ Mor $\Re$. Let $F(X, M)=\{f: X \rightarrow M \mid \forall x \in X f(x) \in$ $\subseteq \underset{y \in X}{\bigcup} M(x, y)\}$, where $M(x, y)$ is the set of morphisms from $x$ into $y$. Let $S$ be a monoid and consider

$$
\mathfrak{U}=\{(s, f) \mid s \in S, f \in F(X, M), \forall x \in X, f(x) \in M(x, s x)\} .
$$

An associative operation is defined on $\mathfrak{A}$

$$
(s, f)(t, g)=(s t, f, g),
$$

where $\left(f_{t} g\right)(x)=f(t x) g(x)$ for all $x \in X$. The semigroup $\mathfrak{A}$ is called the wreath product of $S$ with $\Omega$, and is denoted by $(S: \nu r \Re)$.

It is easy to see that the usual wreath product $(S w r R \mid B)$ of monoids $S$ and $R$ by the left $S$-act $B$ can be considered as $(S w r \Omega)$, where $\Omega$ is such a category that

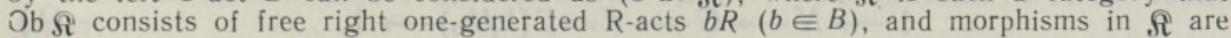
$R$-homomorphisms.

For several classes of categories we prove that $(S \omega r \Omega) \cong(T w r \Omega)$ if $S \cong T$, $\Omega \cong \Omega$. As a corollary we obtain that if $A$ and $B$ are admitted (see $\left[{ }^{8}\right]$ ) left acts over monoids $S$ and $T$, respectively, and $R$ and $P$ are monoids, then

$$
(S \omega r R \mid A) \cong(T w P \mid B) \text { if } S \cong T, R \cong P,|A|=|B| .
$$

This theorem completes the main result of L. A. Skornyakov $\left.{ }^{8}\right]$. 